\title{
A new approach for data transmission system on topological surfaces
}

\author{
JOÃO D. LIMA $^{1 *}$, FRANCISCO M. SILVA ${ }^{1}$ and ELMER R.L. VILLAREAL ${ }^{2}$ \\ ${ }^{1}$ Mestrado em Ciência da Computação, Universidade do Estado do Rio Grande do Norte \\ 59633-010 Mossoró, RN, Brazil \\ ${ }^{2}$ Departamento de Ciências Exatas, Universidade Federal do Semi-Árido \\ 59610-090, Mossoró, RN, Brazil \\ E-mails: jddeus@uol.com.br / miguel.silva@ufersa.edu.br / evillareal@hotmail.com
}

\begin{abstract}
This work introduces a new data transmission system in which the main blocks, coding, modulation and channel are designed on a Riemannian manifolds. An intrinsic algebraic structure to the manifolds (surface), the homology group will be used to compose an error corrector code, a partition on the surface extracted from the embedding of a graph, which will compose the modulation design, and the channel design is the result of an association rule applied to the embedded graph.
\end{abstract}

Mathematical subject classification: Primary: 06B10; Secondary: 06D05.

Key words: modulation, coding, graph embedding, homology group.

\section{Introduction}

Modulation on Riemannian manifold introduced in the 1960s [13], called twister modulation, has been the subject of recent works $[1,9,14]$, due to the good performance presented and of course because of these surfaces which will be the new spaces to be addressed. On a smaller scale, the first proposals for coding on Riemannian manifolds are emerging $[2,8]$. The great source of mathematical results achieved in Riemannian geometry has aroused the interest of researchers in the search of new projects of modulations and codification.

\#CAM-345/11. Received: 13/III/11. Accepted: 23/VIII/11.

*Corresponding author is supported by MCC-UERN-UFERSA. 
In this work, the main components of a data transmission system, the coding, modulation and the channel, will be offered on a Riemannian manifold from an embedding of a graph. As these components are connected by an embedding of a graph, the relation is called compatibility. The data transmission system in which the coding, modulation and channel are compatible among themselves and called of integrated system.

There are many algebraic structures existing in the Riemannian manifolds theory that can be used to compose error corrector codes, however, the homology group is the more natural and is related to the embedded graph [8]. To meet the compatibility relation, we are presenting this structure as a component of a compatible code which attends to the pattern of an integrated system.

The logical principles in which the relations of compatibilities among coding, modulation and channel have been established and their respective geometric constructions that gave rise to the definitions of these components are showed in Section 2. The integrated system definition and a brief comment on the performance are discussed in Section 3. The identification process of compatible modulations is described in Section 4. An example of compatible code, which give information on the integrated system performance is the topic of Section 5. The complete process of the integrated system components' identification will be discussed in Section 6. The conclusions are in Section 7.

\section{Compatibility concepts}

Aiming to introduce the compatibility concepts, it is assumed that a data transmission system uses an alphabet m-ary, indicated by set $\mathbb{Z}_{m}$, where $m$ is a positive integer, to transmit data through a discrete memoryless channel, DMC, with $m$-ary input and $n$-ary output, denoted by $C_{m, n}$. The labeling of the graph vertices, symbols and codewords of this system are represented through a trellis $\Phi$, which section is a sub graph of the complete graph $K_{m, n}$. A particular case of this representation is shown in Figure 1. Note that the sequence $\gamma=$

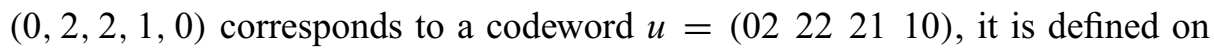
a channel $C_{4,4}$. Thus, $\gamma$ is associated to a connected path of the trellis. The connectivity of the path, results from the fact that a bit information is sent by channel in each interval, condition represented by the trellis. As consequence, 
every source coding sequence or every codeword are represented by a connected path on the trellis.

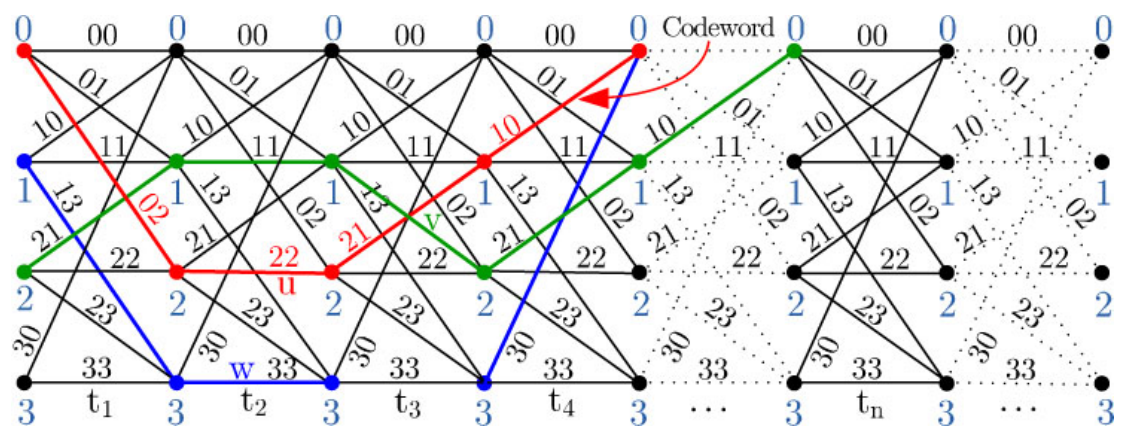

Figure 1 - Trellis corresponding a channel $C_{4,4}$.

Observe that $\gamma_{1}=(2,1,1,2,1,0)$ and $\gamma_{2}=(1,3,3,3,0)$ sequences correspond, respectively, to codewords $v=\left(\begin{array}{lllll}21 & 11 & 12 & 21 & 10\end{array}\right)$ and $w=\left(\begin{array}{lll}13 & 33 & 30\end{array}\right)$, but we can not consider those as codewords coming from trellis $\Phi$. The transition 12 in $v$ is not a trellis edge, and $w$ is not defined on a connected path of the $\Phi$.

\subsection{Compatibility between coding and channel}

In the trellis $\Phi$ are observed two properties: (I) every sequence on the channel $C_{4,4}$ it will be formed by elements of $\mathbb{Z}_{4}$; and (II), if the channel transition of the vertices $i$ to vertices $j$ is represented by $i j$, then $C_{4,4}$ does not have the transitions $03,12,20,31$. Thus, it makes sense to use these two conditions to introduce the concept of compatibility between coding system and channel. To do so, let $\mathbb{Z}_{m}$ be the input of the alphabet and output of the channel $C_{m, m}$.

Definition 2.1. It is said that a coding is compatible with the channel $C_{m, m}$ if every codeword $u$ contains only elements of $\mathbb{Z}_{m}$ and the whole subsequence $i j$ of $u$ is one transition of $C_{m, m}$. Otherwise, we said that coding and channel are incompatible.

If a codeword $u$ satisfies the Definition 2.1, it is easy to see that $u$ is represented by a unique connected path on the $C_{m, m}$ trellis, important condition that establishes the uniqueness of the codewords. The compatibility between 
source coding and channel depends on only the properties (I) and (II). It was observed that linear, blocs and convolutional codes, among others, are important code classes compatible with channels. As it is known that, codewords of linear codes are generated from operations on a generator matrix, then by Definition 2.1, every set of sequences of $\mathbb{Z}_{m}$ associated with connected paths of the trellis are considered a code compatible with a channel $C_{m, m}$. As there are sets that do not come from linear structures, the compatible code classes incorporate other classes of different codes from linear codes. It will be seen later that exists a natural structure of linear code related with this process.

\subsection{Compatibility between modulation and channel}

The natural representative of a discrete memoryless channel $C_{m, n}$ is a bipartite graph $K_{m, n}$ or a sub graph that one. In both cases, a modulation project is necessarily a metrical space partitioned in $n$ decision regions (or Voronoi regions), where signal is present in the center each respective region. One way that such partitions can be obtained is through the dual embedding of 2-cells of bipartite graph $K_{m, n}$. While the embedding condition implies the uniqueness of the channel transitions, the 2-cell embedding implies the uniqueness of the partition. This condition was established by Dyck [4] and later proved by Youngs [12] through the following enunciate.

Theorem 2.2. There is an one-to-one correspondence between rotation system of the graph $G$ and its embeddings on orientable surfaces.

Our purpose is to obtain a modulation project on the metric space that accurately represents the channel transitions and has well defined the Voronoi regions. This is possible if and only if the project is realizable on a surface coming from a graph embedding.

The idea to obtain a modulation project associated with a DMC coming from Lima-Palazzo's work [8]. Later Lima-Lima [7] has showed that is possible to obtain this project through an embedding of a complete graph. A recent analysis in the Lima-Lima's work by Lima-Matias [5], has showed that association process can be simplified and established through any connected graph. It is not a trivial process, it evolves concepts of orientable graph and its dual. 


\subsection{Association process}

The process established by Lima-Lima-Matias consists in taking a connected graph $G_{p, q}$, embedding it on a surface $\Omega$ as 2-cells embedding, constructing the dual embedding, and to define two modulation projects called quadrature amplitude modulations on surface (QAMS). To be more specific, let's suppose that the embedding is of form

$$
G_{p, q} \hookrightarrow \Omega \equiv \cup_{i=1}^{k} R_{\alpha_{i}}
$$

where $R_{\alpha}$ represents a region of $\alpha$ edges defined by sequence $\gamma=\left(\gamma_{1}, \gamma_{2}, \cdots\right.$, $\gamma_{\alpha}$ ) called orbital sequence. In the orbital sequence $\gamma, \gamma_{i} \in\{0,1, \cdots, p-1\}$, corresponds to the vertex $v_{\gamma_{i}}$ of $G$, and $\gamma$ defines the closed path on surface $\Omega$ corresponding the region frontier $R_{a}$. Furthermore, the dual embedding of $G$ is given by $G_{k, q}^{\prime} \hookrightarrow \Omega \equiv \cup_{j=1}^{p} R_{\alpha_{j}}$. As a modulation project for a constellation of $m$ signals might divide the metric space $(\Omega, \delta)$ in $m$ Voronoi regions, in particular, we have two partitions on $\Omega$ : the embedding partition in $k$ regions $\cup_{i=1}^{k} R_{\alpha_{i}}$, and the dual partition in $p$ regions $\cup_{j=1}^{p} R_{\alpha_{j}}$. So, we have two projects of modulation, the modulation $k$-QAMS coming from embedding of $G_{p, q}$, and dual modulation $p$-QAMS. Of course there are modulations for $k$ and $p$ signals, respectively. Once that the signals of the constellation are associated to points of $\Omega, k$ signals of $k$-QAMS correspond to the $k$ vertexes of dual $G^{\prime}$, and $p$ signals of $p$-QAMS correspond to the $p$ vertexes of $G$. Finally, we might associate the channels $C_{k, k}$ and $C_{p, p}$ to modulations $k$-QAMS and $p$-QAMS, process that will be described following.

\subsection{Channel compatible with a modulation}

In the Heawood's description process [11] of the graph embedding $G_{p, q} \hookrightarrow$ $\Omega \equiv \cup_{i=1}^{k} R_{\alpha_{i}}$, the partition $\cup_{i=1}^{k} R_{\alpha_{i}}$ is obtained through the orientable graph (or digraph). In this process, an orientation on vertexes of $G$ defines $k$ regions of partition (Voronoi regions of $k$-QAMS). But on the digraph $G$, each edge $e=\left(v_{i}, v_{j}\right)$ contains its inverse $e^{-1}=\left(v_{j}, v_{i}\right)$, and so, each edge $e=\left(v_{i}, v_{j}\right)$ of $G$ defines two transitions on the channel $C_{k, k}$, and each edge $e=\left(v_{h}, v_{k}\right)$ of dual $G^{\prime}$ defines two transitions on the channel $C_{p, p}$. Thus, we can define a channel $C_{k, k}$ compatible with a modulation $k$-QAMS, for this. 
Let $G_{p, q} \hookrightarrow \Omega \equiv \cup_{i=1}^{k} R_{\alpha_{i}}$ be a 2-cells embedding of a graph $G$, and $G_{k, q}^{\prime} \hookrightarrow$ $\Omega \equiv \cup_{j=1}^{p} R_{\alpha_{j}}$, is a dual embedding. Suppose that $A=\left\{s_{0}, s_{1}, \ldots, s_{k-1}\right\}$ and $A^{\prime}=\left\{s_{0}^{\prime}, s_{1}^{\prime}, \ldots, s_{p-1}^{\prime}\right\}$ are the respectively alphabetic symbols used in the channels $C_{k, k}$ and $C_{p, p}$.

Definition 2.3. It is said that the modulation $k-Q A M S$ ( $p-Q A M S$ ) is compatible with the channel $C_{k, k}\left(C_{p, p}\right)$, if each edge e $=\left(v_{i}, v_{j}\right)\left(e^{\prime}=\left(v_{h}, v_{k}\right)\right)$ of the graph $G\left(G^{\prime}\right)$ defines two transitions $\left(s_{i}, s_{j}\right)$ and $\left(s_{j}, s_{i}\right)\left(\left(s_{h}, s_{k}\right)\right.$ and $\left.\left(s_{k}, s_{h}\right)\right)$ of the channel $C_{k, k}\left(C_{p, p}\right)$.

If a modulation $m$-QAMS and a channel $C_{k, k}$ satisfy the condition of Definition 2.3, it still will said that the channel $C_{k, k}$ is compatible with the modulation $k$-QAMS.

In this process it is has been considered a discrete memoryless channel. The 2-cell embedding can even be used in this kind of project, it is missed only the condition of uniqueness, because exist identical modulations coming from distinct embeddings. But it is impossible discard the embedding condition, therefore, there would not be uniformity of channel transitions. A modulation is the process to broadcast a signal, but here, we are going to consider the modulations as a space metric $(\Omega, d)$ with a region partition $\cup R_{\alpha}$ (where $R_{\alpha}$ is an $\alpha$-gon) on $\Omega$, denoted by $\Xi=\left(\Omega, d, \cup R_{\alpha}\right)$. By Theorem 2.2, the modulation $\Xi$ compatible with channel $C_{m, n}$ is well defined by rotation system of complete graph, $\Theta\left(K_{m, n}\right)$. If the objective is to describe a modulation relating to its all components, then we write

$$
\Xi: K_{m, n}(\Theta) \hookrightarrow \Omega \equiv \cup R_{\alpha} .
$$

A modulation $m$-QAMS is compatible with a channel $C_{m, m}$, when the rotation system $\Theta$ and the 2-cells embedding are fixed. From the algebraic point of view, it is always possible to identify the kind of regions, genus surface and the own partition, but, the topological embedding is highly complex. In this work, it will be seen some non trivial examples.

In Figure 2, trellis section consists in a graph $K_{4,4}(8,9)$ associated to the channel $C_{4,4}$, which the rotation system is given by

$$
\Theta\left(K_{4,4}\right)=\{0(135), 1(062), 2(137), 3(042), 4(357), 5(04), 6(17), 7(264)\} .
$$


(a)

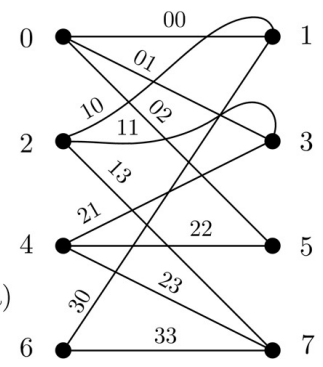

(d)

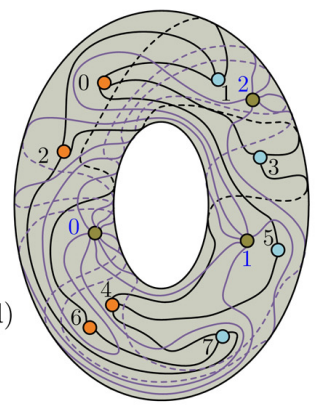

(b)

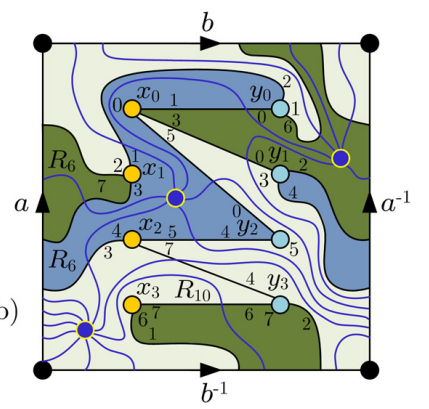

(e)

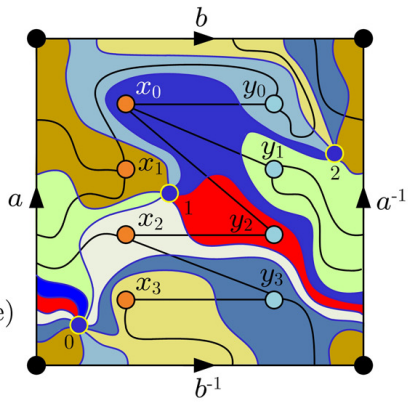

(c)

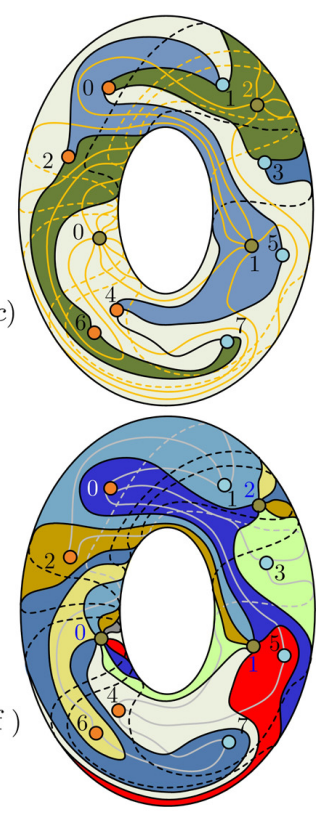

Figure 2 - Steps of a topological modulation projects.

This partition embedding is composed by a single region 22-gon defined by orbital sequence $\gamma=(1,0,3,4,5,0,1,6,7,4,3,2,7,6,1,2,3,0,5,4,7,2)$. It is a maximal embedding on bi-torus of the kind $K_{4,4}(8,9) \hookrightarrow 2 T \equiv R_{22}$. As is not used modulation for one signal, only dual modulation is defined. As $K_{4,4}$ has 8 vertexes, the dual modulation is the form 8-QAMS, each decision region is composed by 1-gon. To better understand this process, we will see the next example. Choosing other rotation system for graph $K_{4,4}(8,9)$, for example,

$$
\Theta^{\prime}\left(K_{4,4}\right)=\{0(135), 1(026), 2(137), 3(024), 4(357), 5(04), 6(17), 7(264)\}
$$

(see Figure 2(a)), we obtain a different embedding $K_{4,4}(8,9) \hookrightarrow T \equiv 2 R_{6}+$ $R_{10}$ defined by sequences $\gamma_{1}(0,3,2,7,6,1), \gamma_{2}(0,5,4,3,2,1)$ and $\gamma_{3}(3,5,4$, $7,2,1,6,7,4)$ (see Figure 2(b-c)). The dual embedding is given by $K^{\prime}(3,9) \hookrightarrow$ $T \equiv 2 R_{2}+6 R_{3}$, and defined by dual $\gamma_{x_{0}}(1,0,2), \gamma_{y_{0}}(1,2,0), \gamma_{x_{1}}(1,0,2)$, $\gamma_{y_{1}}(1,2,0), \gamma_{x_{2}}(1,0,0), \gamma_{y_{2}}(1,0), \gamma_{x_{3}}(0,2)$ e $\gamma_{y_{3}}(0,2,0)$ (see Figure $2(\mathrm{c}-\mathrm{e})$ ).

In Figure 2, embeddings (b) and (c) correspond to the planar and spacial topological projects of modulation 3-QAMS on the torus for a constellation 
of three signals which decision regions are defined by orbital sequences $\gamma_{1}, \gamma_{2}$, $\gamma_{3}$. Its compatible channel $C_{3,3}$ is shown in Figure 3(a). Embeddings (d) and (e) of the Figure 2 correspond to the planar and spacial topological projects of dual modulation 8-QAMS on the torus for a constellation of eight signals which decision regions are defined by orbital sequences $\gamma_{x_{0}}, \gamma_{y_{0}}, \ldots, \gamma_{x_{3}}, \gamma_{y_{3}}$. Its compatible channel $C_{8,8}$ is shown in Figure 3(a). The embeddings of $K_{4,4}(8,9)$ and their dual, shown in Figure 2, represent the complete steps of a topological modulation projects 3-QAMS and 8-QAMS compatible with the channels $C_{3,3}$ and $C_{8,8}$ which associated graph was fixed by rotation in (a). The plane model in (b) illustrates the partition on the torus caused by embedding of $K_{4,4}$ and its dual. The dual graph partition embedding together is shown in (e). The constructions (b) and (d) are very important because they are necessary to construct the modulation on torus, through its parametric equation, and to lead the problem to the Riemann Geometry environment. Finally, (c) and (e) show the spacial models corresponding to the embeddings (b) and (d). The goal of these constructions is to verify that topological modulation is compatible with channel which can be constructed on a topological riamannian manifolds.

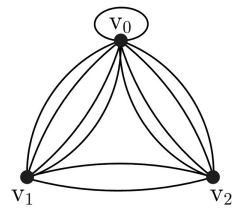

(a) Dual graph $\mathrm{K}^{\prime}$

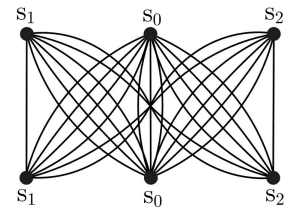

(b) Compatible channel $\mathrm{C}_{3,3}$

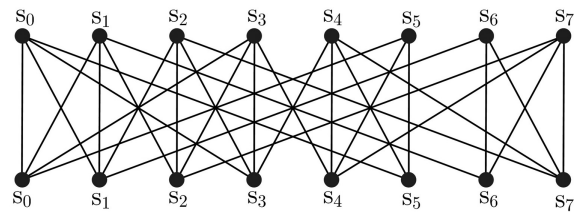

(c) Channel $\mathrm{C}_{8,8}$ compatible with 8-QAMS

Figure 3 - Dual graph and compatible channels.

It is observed that, the channel $C_{k, k}$ compatible with modulation $k$-QAMS is defined on the dual partition from an embedding of graph

$$
G_{p, q} \hookrightarrow \Omega \equiv \cup_{i=1}^{k} R_{\alpha_{i}} .
$$

But $K_{m, n}$ and its dual, are both embeddings in $\Omega$ and is a dual in another. On the other hand, if the dual defines the modulation $p$-QAMS compatible with the channel $C_{p, p}$, this is, defined on the graph $G_{p, q}$. Thus, embedding of $K_{m, n}$ defines a compatible modulation, called directed compatible modulation with $C_{n, n}$. Sometimes we will call dual compatible modulation to refer the compatible modulation coming from the dual embedding. Despite of the compatible 
modulation on the embedding partition is not always uniform, the dual always will be, if the vertices degree of the associated channel is considered satisfying a determined condition.

Proposition 2.4. Let $G(p, q) \hookrightarrow \Omega \equiv \cup_{i=1}^{k} R_{\alpha_{i}}^{i}$ be a 2-cells embedding of graph G. If $C_{k, k}$ and $C_{p, p}$ are DMC channels compatible with modulations $k-Q A M S$ and $p-Q A M S$, then are true the followings affirmations:

(i) For all $k, \gamma\left(G_{p, q}\right) \leq m \leq \gamma_{M}\left(G_{p, q}\right)$, there are directed and dual compatible modulation in $m \Omega$.

(ii) The directed and dual compatible modulations are modulations on $\Omega$ for a constellation of $k$ and $p$ signals, respectively.

(iii) The dual compatible modulation is uniform if, and only if, all vertices of $G_{p, q}$ have the same degree.

Proof. The affirmation (i) follows by Theorem 2.2, (ii) is an embedding partition consequence and dual definition, and (iii) is due the number of edges of one dual region, the vertex degree in its inner, as the uniform modulation, all regions have the same number of the edges.

By Proposition 2.4, it follows that dual modulations coming from complete graph $K_{n}$ and bipartite complete graph of the form $K_{n, n}$, are uniform modulations.

\section{Data transmission integrated system}

In the Definitions 2.1 and 2.3, we have the necessary conditions that we have been looking for to define data transmission system to our interest.

Definition 3.1. We said that a data transmission system is an integrated system (IS), if the coding and the modulation projects are compatible with the channel.

It is still said that in the IS, the coding, modulation and channel are compatible among themselves. In the IS block model shown in Figure 4, the combination $m$-ary input in the modulator, the physical channel, and the $n$-ary output in 
the demodulator are modeled as a discrete memoryless channel (DMC), denoted by $C_{n}$. The modulation design come from the partition of 2-cells embedding of the complete graph $K_{n}$ in the surface $\Omega_{3}$.

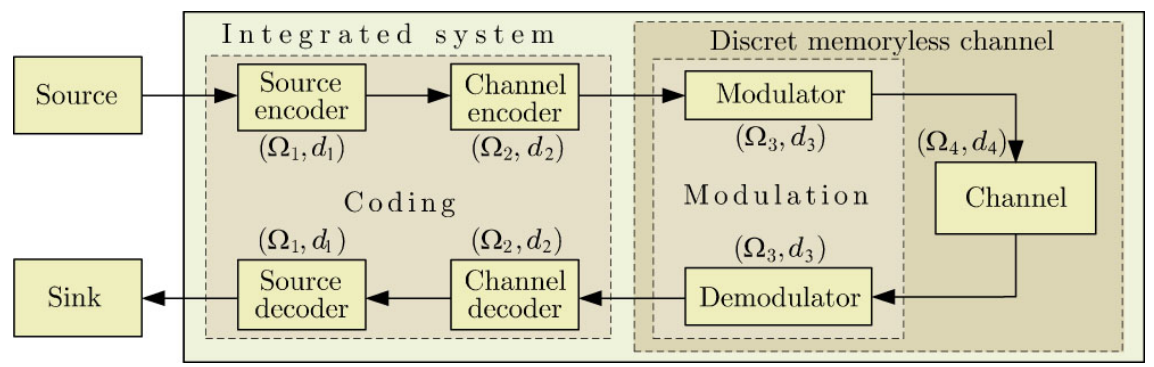

Figure 4 - Integrated system block model.

It's known [8] that the modulation efficiency depends on the genus and uniformity degree $\alpha$ (number of regions of the same kind), additionally, it is shown in [7] that they still depend on the number of edges of the regions. The identification of these parameters is very important in order to know the IS performance. In Figure $5 P_{e}(\alpha)$ indicates the error probability in IS when is used an uniform modulation of degree $\alpha$. In IS, the metric spaces can be considered the same or not [1]. About the compatibility conditions in Definitions 2.1 and 2.3, there are relations one-to-one among coding, modulation and channel, since these are directly related to the channel. This gave us the assurance that IS, in terms of implementation, is the one which has the lowest complexity, since there is no need to introduce additional devices of incompatibility control. Furthermore, someone should consider that the project is done on a Riemannian manifold, space that offers some options for projects with interest properties [1].

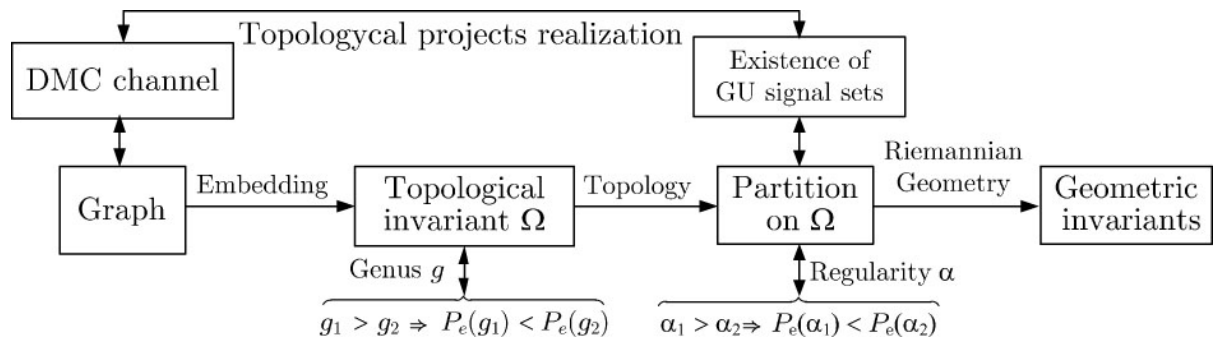

Figure 5 - Proposal overview (Adapted by [1]). 


\section{Identification process of compatible modulations}

We notice the existence not only of a unique compatible modulation with a channel, but a whole compatible modulations set. Although this set was for a finite number of elements, which it grows quickly with increasing signs and channel transitions, it follows the more important topological invariants and results used in the identification process of the IS components.

If $V(G)$ is set of the vertices of $G$ and $\operatorname{deg}(v)$ is the degree of the vertex $v \in V$, then it is easy to verify that graph $G$ has

$$
\prod_{v \in V(G)}(\operatorname{deg}(v)-1) ! \text { rotations. }
$$

If $v, e$ and $f$ are, respectively, the vertex, edges and regions number, then the Eüler's characteristic of the surface $\Omega$ is given by

$$
\chi(\Omega)=v-e+f .
$$

The genus $g$ and $\widetilde{g}$ of the orientable $\Omega$ and non-orientable $\widetilde{\Omega}$ surfaces, in relation to the $\chi(\Omega)$, are given by

$$
\chi(\Omega)=2-2 g \text { and } \chi(\widetilde{\Omega})=2-\widetilde{g} .
$$

Let $\{x\}$ be the smallest integer greater than or equal to the number $x$. The minimal genus of the orientable surface [11] is given by

$$
g(m, n)=\{(m-2)(n-2) / 4\}, \text { for } m \text { and } n \geq 2 .
$$

Let $[x]$ be the greatest integer less than or equal to number $x$. The maximal genus of the orientable surface [10] is given by

$$
\gamma_{M}\left(K_{m, n}\right)=\left[\frac{1}{2}(m-1)(n-1)\right], \text { for } m \text { and } n \geq 2 .
$$

The following theorem [3] gave the orientable surfaces set where is present in the compatible modulation with a channel $C_{m, n}$.

Theorem 4.1. If $G$ has 2-cell embeddings on surfaces of genus $m$ and $n$, then for every integer $k, m \leq k \leq n, G$ has a 2-cell embedding on the surface of genus $k$. 
For example, if we take the bipartite complete graph $K_{4,4}$, what would be modulations coming from embedding of $K_{4,4}$ and their compatible channels? The first step of the identification process is to choose one of the four surfaces families: Non-orientable and orientable with and without component boards. It will be identified modulations for the two cases of surfaces without boards.

\subsection{Compatible modulations on orientable surfaces}

In relation to the orientable without board case, the equality (1) shown that $K_{4,4}$ has 1679616 rotations. Then there are 1679616 options to choose a rotation, but, not all the partitions are distinct, then it is possible to find them. By equalities (4)-(5), it is deducted that minimal and maximal embeddings of $K_{4,4}$ it has been found in surfaces of genus $\gamma=1$ and $\gamma_{M}=4$. So, by Theorem 4.1, orientable surfaces set to the $K_{4,4}$ embeddings is given by $\mathbb{S}\left(K_{m, n}\right)=\{T, 2 T, 3 T, 4 T\}$. On the other hand, $K_{4,4}$ has 16 edges, the regions of the $K_{4,4} \hookrightarrow \Omega$ have 32 edges and embedding regions of $K_{4,4}$ only contain an even number of the edges larger than or equal to four. These affirmations conclude that 2-cell embeddings of $K_{4,4}$ must necessarily be listed in the Table 2, where partition of $m T$ is the same of $2 m P$. The notation $R_{a_{1}, a_{2}, \cdots, a_{k}}$ used in Table 2 indicates the embedding partition of $K_{m, n}$ in $k$ regions with $a_{1}, a_{2}, \cdots, a_{k}$ edges.

An attribute of great interest in a modulation is its uniformity or, equivalently, the condition: Voronoi's regions are all congruent. Condition equivalent to the geometrically uniform signal space, here, correspondents to the embeddings with regions of the same kind, as examples, the partitions $8 R_{4}$ of $T$ and $4 R_{8}$ of $3 T$.

The Theorem 4.1 gave us the guarantee for the existence of, at least, an embedding with element of $\mathbb{S}$. Soon, we do not have doubt that the minimal embedding $K_{4,4} \hookrightarrow T \equiv 8 R_{4}$ exists, an example of uniform compatible modulation. We still check the existence of the regular embedding $K_{4,4} \hookrightarrow 3 T \equiv 4 R_{8}$, other important example of regularity. These examples illustrated in Figure 6 shows that $K_{m, n}$ embeddings are a rich source of uniform compatible modulation. It is observe that directed and dual modulations coming from minimal embedding $K_{4,4}$ are equals. This one is an uniform modulation for a constellation of 8 signals, with Voronoi's regions of the kind 4-gon. Its dual graph and compatible 


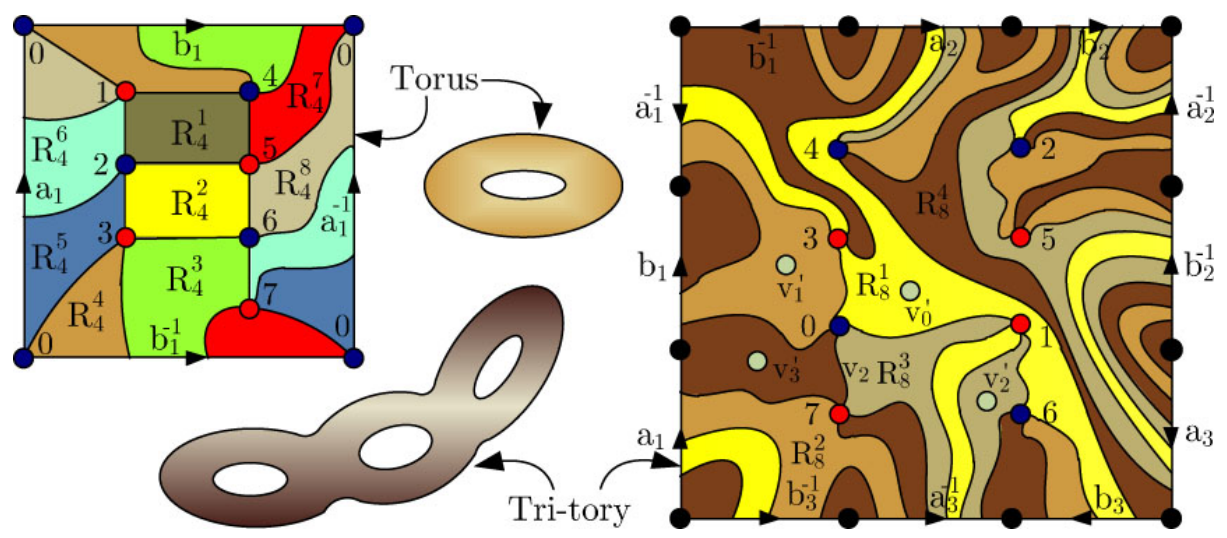

Figure 6 - Uniform modulations QAMS on the torus and 3-tory coming from $K_{4,4}$ embeddings.

channel is the same kind of channel in Figure 7(c). And 3-tory one, directed compatible modulation have 4 signals, with Voronoi's regions of the kind 8-gon. Dual graph (a) and compatible channel (b) is shown in Figure 7. Dual compatible modulations are uniform modulations for a constellation of 8 signals with decision regions of the kind 4-gon (see its channel in (c)). It is showing that geometrically uniform signal space is not privilege only of hyperbolic spaces.

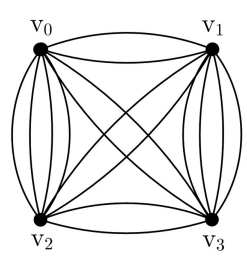

(a) Dual Graph $\mathrm{K}_{4}^{\prime}$

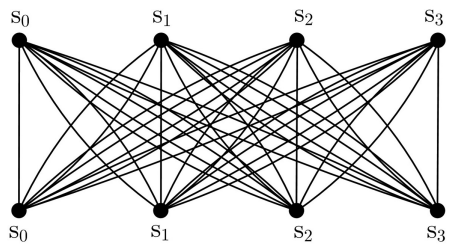

(b) Channel $\mathrm{C}_{4,4}$ compatible with 4-QAMS

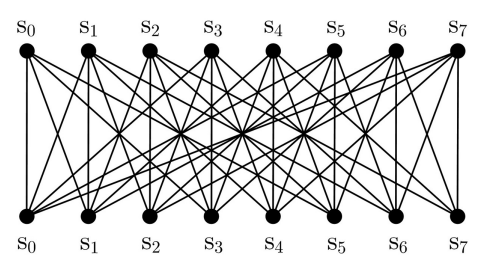

(c) Channel $\mathrm{C}_{8,8}$ compatible with 8-QAMS

Figure 7 - Dual graph and compatible channels with the uniform modulations on $3 T$ in the Figure 6.

An embedding of a graph $G$, and thus, its compatible modulations, are determined by a rotation system $\Theta$ of $G$, dual embedding, and sets of orbital sequences of embeddings of $G$ and dual $G^{\prime}$. This process can be realized only with algebraic resource, using a algorithm descriptor of embedding [6]. (See algebraic description process in Table 1). 


\begin{tabular}{|c|l|l|c|}
\hline$\Omega$ & \multicolumn{1}{|c|}{ Rotation } & \multicolumn{1}{c|}{ Orbital sequence $\gamma_{i}$} & $\Xi$ \\
\hline \multirow{2}{*}{$T$} & $(1573)_{0},(0426)_{1},(1537)_{2},(0264)_{3}$, & $(1254)_{1},(2365)_{2},(3476)_{3},(0143)_{4}$ & \multirow{2}{*}{$8 R_{4}$} \\
& $(1375)_{4},(0624)_{5},(1735)_{6},(0462)_{7}$ & $(0327)_{5},(1672)_{6},(0745)_{7},(0561)_{8}$ & \\
\hline \multirow{2}{*}{$3 T$} & $(1753)_{0},(0642)_{1},(1375)_{2},(0642)_{3}$ & $1(30163412), 2(03654725)$ & \multirow{2}{*}{$4 R_{8}$} \\
& $(1573)_{4},(0642)_{5},(1357)_{6},(0642)_{7}$ & $3(10761452), 4(70567432)$ & \\
\hline
\end{tabular}

Table 1 - Uniform compatible modulations with $C_{4,4}$.

In Table 1, the notation (1573) $)_{0}$ used in second column corresponds to the rotation of the vertex $0,(1254)_{1}$ used in the $3^{r d}$ column indicated the orbital sequence $\gamma_{1}$ on the torus, and $\Xi$ indicated the partition. It was verified even that the $R_{6,6,8,10}$ is the unique partition of the Table 2 non existent in one orientable embedding of $K_{4,4}$ [5]. It would be this partition in a non orientable embedding of $K_{4,4}$ ?

\subsection{Irregular compatible modulation with $C_{4,4}$}

In Table 2, most of the embeddings of $K_{4,4}$ are irregulars. Using the embedding method through homology curves incises [8], plane (b) and space (a) models of a compatible modulations with $K_{4,4}$ are illustrated in Figure 8. This is an embedding of the form $K_{4,4} \hookrightarrow 3 T \equiv R_{4,6,6,16}$ (see plane model in (a)). It can be used as a non-uniform directed compatible modulation project for constellation of 4 signals, where decision regions $R_{4}, R_{6}, R_{6}, R_{16}$ correspond orbital sequences $\gamma_{1}$ (4563), $\gamma_{2}(052743), \gamma_{3}(012367), \gamma_{4}(1032541650721476)$, respectively, and an uniform dual compatible modulation for constellation of 8 signals coming from dual partition $K_{4,4} \hookrightarrow 3 T \equiv 8 R_{4}$ (see embedding (e)), witch decision regions are defined by $\gamma_{1}(4243), \gamma_{2}(4434), \gamma_{3}(4432), \gamma_{4}(1324)$, $\gamma_{5}(1244), \gamma_{6}(1424), \gamma_{7}(1443), \gamma_{8}(2434)$.

It is concluded, therefore, that each embedding of $K_{4,4}$ defines two compatible modulations: that directly coming from the embedding partition and the dual partition. Despite of the compatible modulation on the embedding partition of the graph $G$ is not always uniform, the dual always will be, if the vertices degree of $G$ are equals. For example, when $G$ is the complete graph or bipartite complete graph. 

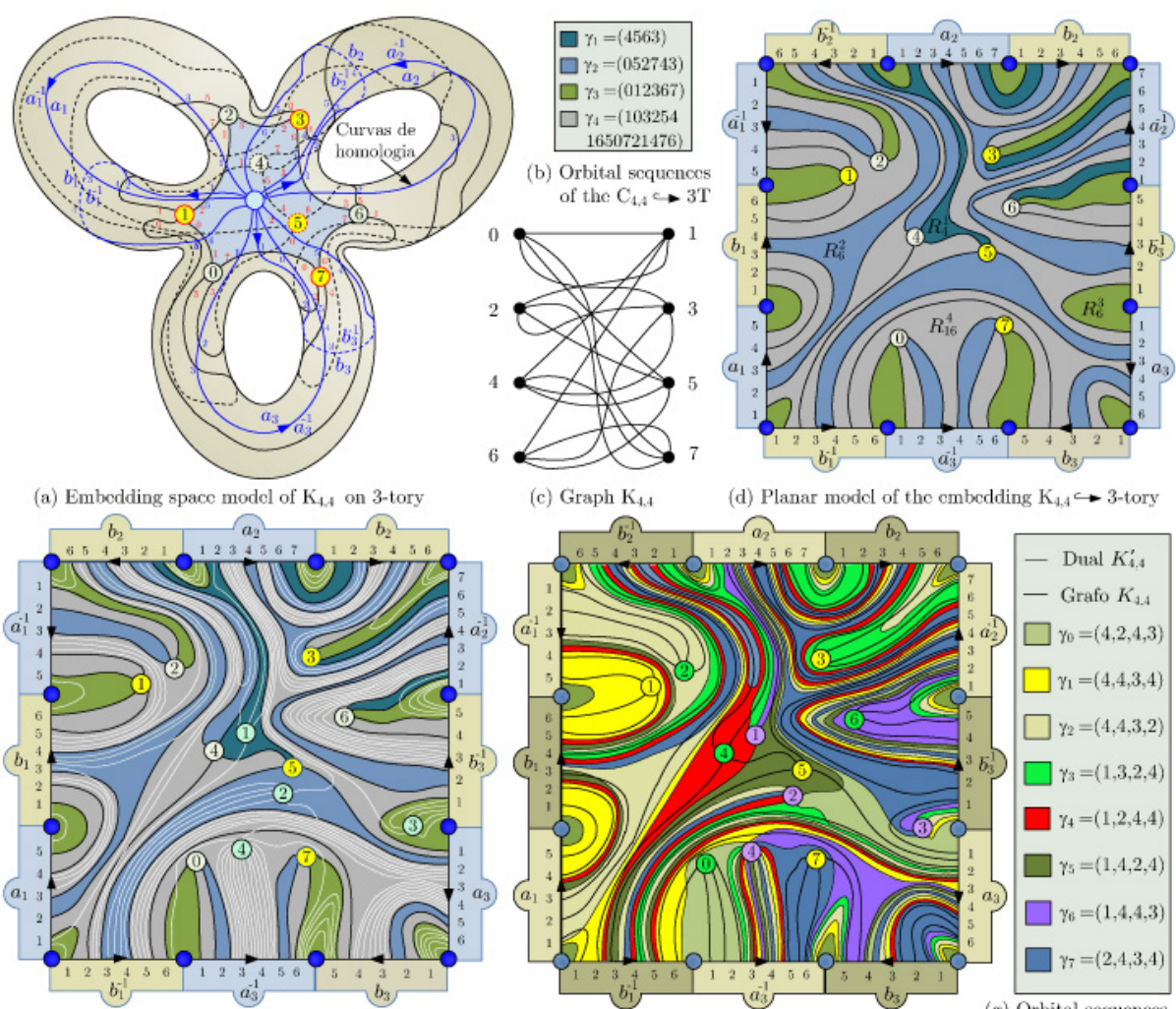

(c) Graph $\mathrm{K}_{4,4}$

(d) Planar modd of the cmbedding $\mathrm{K}_{4,4} \hookrightarrow$-tory

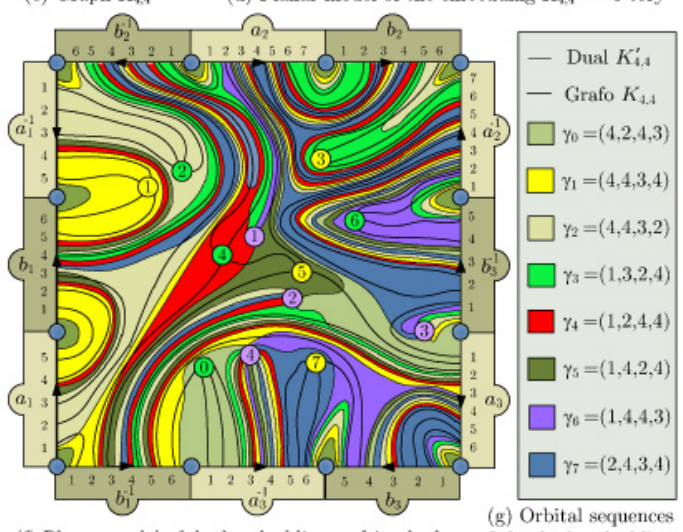

(e) Planar model of embding $\mathrm{K}_{4,4} \hookrightarrow$-tory and its dual

(f) Planar model of dual embedding and its dual

of the dual embedding

Figure 8 - Irregular modulations in 3-tory.

\subsection{Compatible modulation on non-orientable surfaces}

The identification process of modulation on non-orientable surfaces is similar to the orientable case. The difference is the variation of Eüler characteristic, it is of two in two unities in the orientable case and one to one it is the nonorientable case. As consequence, there are partitions in non-orientable surfaces that are not found in an orientable embeddings.

An orientable surface has been represented by a connected sum of $g$ tory, denoted by $g T$. Similarly, a non-orientable surface it will be indicated by a connected sum of $\tilde{g}$ projective planes $P$, denoted by $\tilde{g} P$. Taking into account the fact that the embedding of $K_{4,4}$ only has regions of the kind $2 k$-gon, $k \geq 2$, and sum of the region edges is 32 , it is concluded that directed compatible 
modulations with the channel $C_{4,4}$ in non-orientable surface are as the related models in Table 2.

\begin{tabular}{|c|l|c|}
\hline$\tilde{g} P$ & \multicolumn{1}{|c|}{ Partitions } & $\mathrm{N}^{0}$ \\
\hline $2 P$ & $R_{4,4,4,4,4,4,4,4}$ & 1 \\
\hline $3 P$ & $R_{4,4,4,4,4,4,8}, R_{4,4,4,4,4,6,6}$ & 2 \\
\hline $4 P$ & $R_{4,4,4,4,4,12, R_{4,4,4,4,6,10}, R_{4,4,4,4,8,8}, R_{4,4,4,6,6,8}, R_{4,4,6,6,6,6}}$ & 5 \\
\hline $5 P$ & $\begin{array}{l}R_{4,4,4,4,16}, R_{4,4,4,6,14}, R_{4,4,4,8,12}, R_{4,4,4,10,10}, R_{4,4,6,6,12} \\
R_{4,4,6,8,10}, R_{4,4,8,8,8}, R_{4,6,6,6,10}, R_{4,6,6,8,8}, R_{6,6,6,6,8}\end{array}$ & 10 \\
\hline $3 T$ & $\begin{array}{l}3 R_{4} R_{20}, R_{4,4,6,18}, R_{4,4,8,16}, R_{4,4,10,14}, R_{4,4,12,12}, R_{6,6,6,14}, R_{6,6,8,12}, 4 R_{8} \\
R_{4,6,6,16}, R_{4,6,8,14}, R_{4,6,10,12}, R_{4,8,8,12}, R_{4,8,10,10}, R_{6,6,10,10}, R_{6,8,8,10}\end{array}$ & 15 \\
\hline $7 P$ & $\begin{array}{l}R_{4,4,24}, R_{4,6,22}, R_{4,8,20}, R_{4,10,18}, R_{4,12,16}, R_{4,14,14}, R_{8,12,12} \\
R_{6,8,18}, R_{6,10,16}, R_{6,12,14}, R_{8,8,16}, R_{8,10,14}, R_{6,6,20}, R_{10,10,12}\end{array}$ & 14 \\
\hline $8 P$ & $R_{4,28}, R_{6,26}, R_{8,24}, R_{10,22}, R_{12,20}, R_{14,18} R_{16,16}$ & 7 \\
\hline $9 P$ & $R_{32}$ & 1 \\
\hline
\end{tabular}

Table 2 - Directed compatible modulations coming from $K_{4,4}$ embeddings on non-orientable surfaces.

Observe that partitions of non-orientable embeddings are always the same of the orientable embeddings always $\widetilde{g}$ is an even number, and in the cases where $\widetilde{g}$ is odd, the number of partitions is still quickly greater than the orientable partitions. So the non-orientable embeddings of $K_{4,4}$, when compared to orientable case, is a modulation source with more than double of elements of the orientable case, can have the same kind of partitions and present different options of embeddings and regularities. In relation to topological projects, these can be constructed with same difficulty degree of orientable embeddings, as shown the Figure 9. A compatible directed modulation with the channel $C_{12,12}[4,4]$ is illustrated in (a). In the graph $K_{6,6}, \operatorname{deg} v=4$ and rotation is given by

$$
\begin{aligned}
\Theta= & \{0(1,3,11,9), 1(0,10,2,4), 2(1,11,9,5), 3(0,4,6,8), \\
& 4(1,5,7,3), 5(2,6,8,4), 6(3,7,9,5), 7(4,8,10,6), 8(5,3,11,7), \\
& 9(6,10,0,2), 10(9,7,11,1), 11(10,8,0,2)\} .
\end{aligned}
$$

The plane model in (b) and spacial model in (c) represent homeomorphic embeddings corresponding to the partition $K_{6,6}(12,24) \hookrightarrow 2 P \equiv 12 R_{4}$, is so 

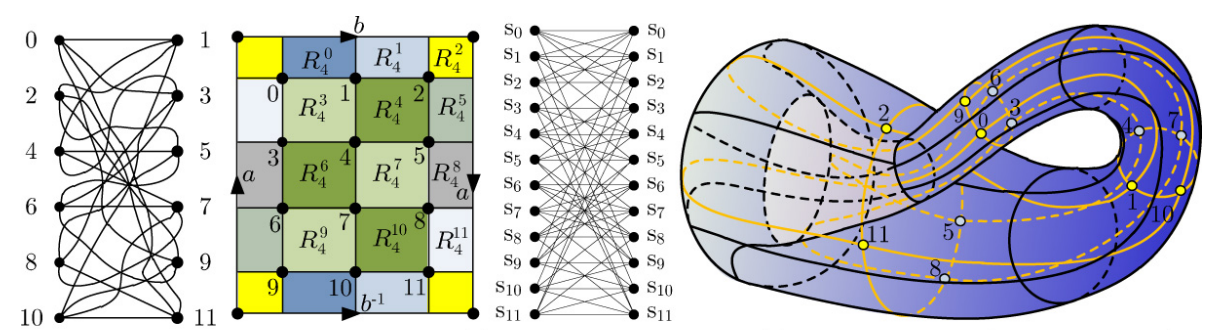

(a) Graph $\mathrm{C}_{6,6}[4,4]$ (b) $\mathrm{C}_{6,6} \hookrightarrow \mathrm{K} \equiv 12 \mathrm{R}_{4}$ (c) Compatible Channel $\mathrm{C}_{12} \quad$ (d) $\mathrm{C}_{6,6} \hookrightarrow \mathrm{K} \equiv 12 \mathrm{R}_{4}$ (space embedding)

Figure 9 - Compatible modulation with channel $C_{12}$ coming from $K_{6,6}[4]$ on the surface Klein's Bottle.

an example of a compatible uniform modulation on a non-orientable surface of genus 2, the Klein's bottle, of the same homeomorphis class of the surface $2 P$. As the dual is equal to the own embedding, directed and dual compatible modulation are identical. This is a very important example because it is possible to be realized, a Differential Geometry level, through its parametric equations.

\section{Homology group and compatible code}

It has been seen that a compatible code with a DMC can be taken in different ways. The more natural way to do this choice [8] is that it should be through the homology group. The reason is because this group can be determined, when the Betti's method is applied from a triangulation obtained from a graph embedding. Thus, the proposal is to establish a compatible linear code extracted directly from the calculus process of homology group via Betti's method. In this sense, it is known that homology group of orientable and non-orientable surfaces is given by

$$
H_{1}(\Omega)=\left\{\begin{array}{l}
\mathbb{Z}^{2 m}, \text { if } \Omega \equiv m T \\
\mathbb{Z}_{2} \oplus \mathbb{Z}^{m-1}, \text { if } \Omega \equiv m P,
\end{array}\right.
$$

where $\mathbb{Z}$ is the additive group of the integers, $\mathbb{Z}_{2}$ is the binary group and $\mathbb{Z} \oplus \mathbb{Z}$ is a directed sum of $\mathbb{Z}$ with its own.

A strong reason for choice the homology group structure as an error-correcting code provider, beyond the uniqueness of this one, are the facts of the 2-cells embedding introduce a unique modulation and the rotation system provide a unique channel, conditions in which the own IS is determined by a unique way. 
Moreover, the Betti's method determines the homology group through linear system which is associated to the matrix that can be used as an error-correcting code generator, similarly to linear codes. The idea is to identify and analyze the correct error capacity of these structures.

Suppose the graph $K_{m, n}$ is embedding on the surfaces $2 T$ and $4 P$, as a 2-cells embeddings. It is easy to construct the triangulation on these embeddings. Thus, let $\Upsilon$ be a triangulation or a simplicial complex on $\Omega$. But $\Upsilon$ is a triangulation with many edges, which makes the homology group calculation more laborious. As the homology group is independent of the triangulations $\Upsilon$, this calculus can be minimized using the central triangular diagrams (CTD) [8]. For surfaces $2 T$ and $4 P$ the CTD are shown in Figure 10.

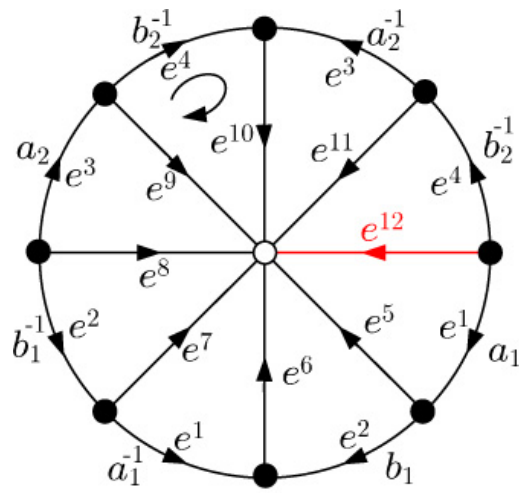

(a) $2 T \equiv a_{1} b_{1} a_{1}^{-1} b_{1}^{-1} a_{2} b_{2} a_{2}^{-1} b_{2}^{-1}$

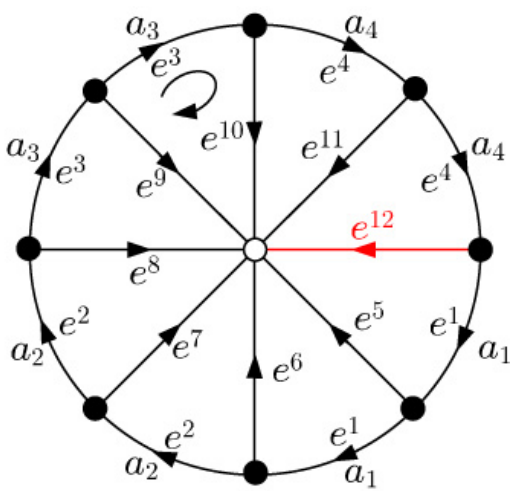

(b) $4 P \equiv a_{1} a_{1} a_{2} a_{2} a_{3} a_{3} a_{4} a_{4}$

Figure $10-$ Central triangular diagrams on $2 T$ and $4 P$.

From Betti's method, the first homology group, indicated by $H_{1}(\Omega)$, of the surfaces $2 T$ and $4 P$ are given, respectively, by solutions of the linear systems

$$
H(2 T):\left\{\begin{array}{l}
z_{1}+z_{2}+z_{6}=0 \\
z_{1}+z_{6}-z_{7}=0 \\
z_{2}+z_{7}-z_{8}=0 \\
z_{3}-z_{8}+z_{9}=0 \\
z_{3}+z_{4}+z_{10}=0 \\
z_{4}-z_{9}+z_{10}=0
\end{array} \quad, H(4 P):\left\{\begin{array}{l}
2 z_{1}+z_{6}=0 \\
z_{2}+z_{7}-z_{6}=0 \\
z_{2}+z_{8}-z_{7}=0 \\
z_{3}+z_{9}-z_{8}=0 \\
z_{3}+z_{10}-z_{9}=0 \\
z_{10}-2 z_{11}=0
\end{array}\right.\right.
$$


and using the fields $\mathbb{Z}_{2}$ and $\mathbb{Z}_{3}$ it follows the matrices

$$
\begin{aligned}
G= & {\left[\begin{array}{lllllllll}
1 & 1 & 0 & 0 & 1 & 0 & 0 & 0 & 0 \\
0 & 1 & 0 & 0 & 0 & 1 & 0 & 0 & 0 \\
0 & 0 & 1 & 0 & 0 & 0 & 1 & 1 & 0 \\
0 & 0 & 0 & 1 & 0 & 0 & 1 & 1 & 1
\end{array}\right], } \\
\widetilde{G} & =\left[\begin{array}{lllllllll}
1 & 0 & 0 & 2 & 0 & 0 & 0 & 0 & 0 \\
0 & 1 & 0 & 0 & 2 & 1 & 0 & 0 & 0 \\
0 & 0 & 1 & 0 & 0 & 0 & 2 & 1 & 0 \\
0 & 0 & 0 & 1 & 0 & 0 & 0 & 0 & 1 \\
0 & 0 & 0 & 0 & 1 & 1 & 1 & 0 & 0 \\
0 & 0 & 0 & 0 & 0 & 0 & 1 & 1 & 1
\end{array}\right] .
\end{aligned}
$$

Therefore, $G(\Omega)$ is a binary matrix and $G(\widetilde{\Omega})$ is a 3-ary matrix. These properties will be used to obtain the linear codes in orientable and non-orientable surfaces, coming from homology group, with algebraic structures in the fields $\mathbb{Z}_{2}$ and $\mathbb{Z}_{3}$ and patterns of a IS. Then, considering $G(2 T)$ as a generated matrix of an error-correcting code, it has a binary linear code $\left(C_{1}, 4,9\right)$ with source sequences and codewords of lengths 4 and 9 , respectively. Similarly, it is deduced that matrix $G(4 P)$ generates a 3 -ary linear code $\left(C_{2}, 6,9\right)$. These structures of codes are formalized bellow.

Definition 5.1. Let $K_{m, n} \hookrightarrow \Omega$ a 2-cells embedding of the graph $K_{m, n}$, and $\Xi(\Omega)$ the CDT on $\Omega$. It is called compatible generalized code, the linear code $(H, s, t)$ generated by matrix $G(\Omega)_{s \times t}$, obtained from linear system $H_{1}(\Omega)$.

The demands imposed are: the linear independence of the $G(\Omega)$ lines and, to effect of correcting errors, the lines corresponding to the variables $z_{2 g+2}$ e $z_{6 g-4}$ are eliminated. Of course, these last conditions can be refused since they are not lost on condition of linear independence. The parameter $s$ tells us the source code is a linear subspace of the space $\mathbb{Z}_{2}^{s}$ with $2^{s}$ elements and sequences of lengths $s$. The parameter $t$ indicated the code is a linear subspace of the space $\mathbb{Z}_{2}^{t}$ with $2^{t}$ elements and codewords of lengths $t$.

Remark 5.2. If $\Omega$ is a compact surface of genus $g$, then $2 g$-gon $\equiv \Omega$, and all vertices of the boundary of the orientable polygon $2 g$-gon are transformed into 
a single vertex, after the geometric realization of $2 g$-gon in $\Omega$. This property is essential to proof the follow affirmation.

Proposition 5.3. The CDT $\Xi(\Omega)$ satisfies the following properties:

a) The maximal tree on $\Xi(\Omega)$ is composed by a unique 1-simplex (red edges in Figure 10).

b) $\Xi(\Omega)$ is composed by $6 g$ 1-simplexes, if $\Omega \equiv g T$ or $\Omega \equiv \tilde{g} P$ where $\tilde{g}=2 g$.

c) The system of the homology group presentation $H_{1}(\Omega)$, from $\Xi(\Omega)$ is composed by $4 \mathrm{~g}$ trivial relations, being two of two terms and the rest of three terms.

d) The system $H(\Omega)$ contains $4 g-2$ trivial relations and $6 g-2$ variables.

e) $H(g T)$ has two equivalent lines to the trivial relations $z_{2 g+4} \equiv 0$ and $z_{6 g-4} \equiv 0$.

f) The rank of the matrix $G(\Omega)$ is $2 g$ if $\Omega \equiv g T$ and $\widetilde{g}-2$ if $\Omega \equiv \widetilde{g} P$, $\tilde{g}=2 g$.

Proof. Suppose that the maximal tree $A$ has another 1-simplex $e^{j}$ different of $e^{12}$. If $e^{j}$ is an edge on the boundary of the $2 n$-gon, then $e^{j}$ is a loop of $\Omega$. If $e^{j}$ is a ray of the $2 n$-gon, then, by Remark $5.2, e^{j}$ and $e^{12}$ have two interceptions, in the center and on the boundary, and so $A$ has a loop. Both on this case, $A$ would not be a tree, which proofs the affirmation a). As $\widetilde{g}=2 g$, then, both the surfaces $g T$ and $2 g P$ present polygonal forms $2 g$-gon. So its respective CDT $\Xi(\Omega)$ has $g$ different 1-simplexes on the boundary and $2 g 1$-simplexes in the interior of $2 g$-gon, a total of $6 g$ 1-simplexes, which proves $b$ ). In the Betti's method, each trivial relation $r_{i}$ comes from a 2-simplex or triangle $t_{i}$, and by one property known [8], the number of the $r_{i}$ terms are equal to the number of 1 -simplex in $t_{i}$ that does not belong to maximal tree $\Xi(\Omega)$. As only there are two 2-simplexes of $\Xi(\Omega)$ has 1-simplex on $A$, it follows affirmation c). The number of system variables $H(\Omega)$ is equal to the number of 1 -simplex, i.e., $6 g$ and $H(\Omega)$ is obtained replacing the two trivial relations of two terms in two of three terms. As each replacement missed one variable, then $H(\Omega)$ is formed by $4 g-2$ trivial relations and $6 g-2$ variables, which proof d). The affirmation e) 
was checked during the resolution of homology groups, including the solution on CDT of a generic surface. In particular case of $H(2 T)$, he have

$$
\left[\begin{array}{rrrrrrrrrr}
1 & 1 & 0 & 0 & 0 & 1 & 0 & 0 & 0 & 0 \\
1 & 0 & 0 & 0 & 0 & 1 & -1 & 0 & 0 & 0 \\
0 & 1 & 0 & 0 & 0 & 0 & 1 & -1 & 0 & 0 \\
0 & 1 & 0 & 0 & 0 & 0 & 0 & -1 & 1 & 0 \\
0 & 0 & 1 & 1 & 0 & 0 & 0 & 0 & 0 & 1 \\
0 & 0 & 0 & 1 & 0 & 0 & 0 & 0 & -1 & 1
\end{array}\right] \Rightarrow\left[\begin{array}{lllllllrrr}
1 & 1 & 0 & 0 & 0 & 1 & 0 & 0 & 0 & 0 \\
0 & 1 & 0 & 0 & 0 & 0 & 1 & 0 & 0 & 0 \\
0 & 0 & 0 & 0 & 0 & 0 & 0 & -1 & 0 & 0 \\
0 & 0 & 1 & 0 & 0 & 0 & 0 & -1 & 1 & 0 \\
0 & 0 & 0 & 1 & 0 & 0 & 0 & -1 & 1 & 1 \\
0 & 0 & 0 & 0 & 0 & 0 & 0 & -1 & 0 & 0
\end{array}\right]
$$

Notice that the $3^{\text {th }}$ and $4^{\text {th }}$ lines in the second matrix above are identical and both indicate that $z_{8}=0$. For the coding purpose, this type of line does not cause any effect, therefore their exclusion, amongst with variables which contain the null columns in the composition of the generator matrix of compatible code. Therefore, the generator matrix of compatible code on $2 T$, instead of $6 \times 10$, it is a matrix of order $4 \times 9$ (in $\mathbb{Z}_{2}$, it is equal to the matrix $G$ above). The affirmation $\mathrm{f}$ ) result of the equality (6), a classic theorem of the homology groups of the algebraic topology.

Constructing the DTC for generic surface $\Omega$ and its corresponding generate matrices $G(\Omega)$, it is possible to conclude that, for all embedding $K_{m, n} \hookrightarrow \Omega$, exists a unique generalized code compatible $G$ on a Riemann manifold $\Omega$ which the graph associated to the channel $C_{m, n}$ has a 2-cells embedding.

Theorem 5.4. If $K_{m, n} \hookrightarrow \Omega \equiv \cup_{i=1}^{k} R_{\alpha_{i}}^{i}$ is a 2-cells embedding, then exists a unique binary generalized code $H$, if $\Omega \equiv g T$, or a unique 3-ary generalized code $\widetilde{H}$, if $\Omega \equiv g T$, compatibles with $C_{k}$, defined by following parameters,

$$
H=\left\{\begin{array}{l}
(H, 4 g-4,6 g-3), \text { if } \Omega \equiv g T \\
(\widetilde{H}, 2 \widetilde{g}-2,3 \widetilde{g}-3), \text { if } \Omega \equiv \widetilde{g} P .
\end{array}\right.
$$

Proof. By Definition 5.1, the generate matrix $G(\Omega)$ of code $H$ coming from a matrix $H(\Omega)$ associated to the coefficients of a simplified system of trivial relations with three terms, obtained from the presentation of the homology group of $\Omega$, through Betti's method. As $G(\Omega)$ is obtained through fundamental operations, then $G(\Omega)$ is equivalent to $H(\Omega)$ and so the code generated by $G(\Omega)$ is a unique binary code. If $\Omega \equiv g T$, by Proposition 5.3(d), $H(\Omega)$ has $2 g-2$ trivial 
relations and and $6 g-2$ variables. As $G(\Omega)$ is the matrix of the coefficients of $H(\Omega)$ by eliminating two from the line of this one, it following that $G(\Omega)$ is a binary matrix of order $(4 g-4) \times(6 g-3)$. In an analogous way, it is conclude that $G(\widetilde{g} P)$ has a 3-ary matrix of order $(2 g-2) \times(3 g-3)$. As the order $s \times t$ of $G(\Omega)$ defines the parameters $(H, s, t)$ of a linear code, which proves the equalities (8).

Corollary 5.5. Dual code of a generalized code compatible with a channel $C_{m+n}$ is of the form:

$$
H^{\prime}=\left\{\begin{array}{l}
\left(H^{\prime}, 2 g+1,6 g-3\right), \text { if } \Omega \equiv g T \\
\left(\widetilde{H}^{\prime}, \widetilde{g}-1,3 \widetilde{g}-3\right), \text { if } \Omega \equiv \widetilde{g} P
\end{array}\right.
$$

Proof. Directed consequence of the dual code and Theorem 5.4.

As examples, the generalized codes $\left(H_{1}, 4,9\right)$ e $\left(H_{2}, 6,9\right)$ above, relatives the generated matrices $G(2 T)$ e $G(4 P)$, have as dual codes $\left(H_{1}, 5,9\right)$ e $\left(H_{2}, 3,9\right)$, respectively.

\section{Identification process of IS components}

In the case of bipartite complete graph, the main components of IS, the modulations, coding and channel, are obtained through the followings steps.

\subsubsection{Surface sets}

Equalities (4) given the genus $\gamma$ of the minimal orientable embedding of $K_{m, n}$. (The minimal non-orientable embedding $\sim$ rsatisfies the condition $\tilde{\gamma}=\gamma$ or $\tilde{\gamma}=\gamma+1$ ). Thus, by Theorem 4.1, the orientable and non-orientable surface sets for embeddings of $K_{m, m}$ are given by

$$
\begin{aligned}
& \mathbb{S}(\Omega)=\left\{\gamma T,(\gamma+2) T,(\gamma+4) T, \ldots, \gamma_{M} T\right\} \\
& \mathbb{S}(\widetilde{\Omega})=\left\{\tilde{\gamma} P,\left(\sim_{\mathcal{H}} 1\right) P,\left(\sim_{\mathcal{H}} 2\right) P, \ldots, \tilde{\gamma}_{M} P\right\},
\end{aligned}
$$

where $\gamma_{M}$ and $\sim_{N}$ are the orientable and non orientable maximal genus of $K_{m, n}$. 


\subsubsection{Modulation models}

It is determined the Eüler characteristic $\chi(\gamma T)$ by equalities (3) and the region number $\tau$ of the minimal embedding (equalities (2)). As the regions of a $K_{m, n}$ embedding partition have $2 m n$ edges and one region is the form $R_{2 k}$, $k \geq 2$, the modulation models are composed by all possible combinations of partitions $\cup_{i=1}^{t} R_{\alpha_{i}}^{i}$ such that

$$
t= \begin{cases}\tau, \tau+2, \tau+4, \ldots, 5,3,1, & \text { if } t \text { is odd } \\ \tau, \tau+2, \tau+4, \ldots, 6,4,2, & \text { if } t \text { is even. }\end{cases}
$$

In the non-orientable case, is determined the partitions $\cup_{i=1}^{\widetilde{t}} R_{\alpha_{i}}^{i}$ such that $\tilde{t}=\tilde{\tau}, \tilde{\tau}+1, \tilde{\tau}+2, \ldots, 3,2,1$, where $\tilde{\tau}$ is the region number of the nonorientable minimal embedding.

\subsubsection{Compatible code}

Equalities (8) given an algebraic structures for composition of correcting-code error, Theorem 5.4 provides the generalized code compatible with channel $C_{m, n}$, and Corollary 5.5 provides the dual code.

As an application of the method we consider the IS components compatible with channel $C_{4,4}$ identified in the Table 3 . The elements of the associated graph $K_{4,4}$ are: $v=8$ and $e=16$.

\begin{tabular}{|c|c|c|c|c|c|c|}
\hline$g(\Omega)$ & $\Omega$ & $\alpha$ & $N^{o}$ & $H_{1}(\Omega)$ & Code & Dual \\
\hline \multirow{5}{*}{$g T$} & $T$ & 8 & 1 & $\mathbb{Z}^{2}$ & $(H, 1,3)$ & $\left(H^{\prime}, 2,3\right)$ \\
\cline { 2 - 7 } & $2 T$ & 6 & 4 & $\mathbb{Z}^{4}$ & $(H, 4,9)$ & $\left(H^{\prime}, 6,9\right)$ \\
\cline { 2 - 7 } & $3 T$ & 4 & 13 & $\mathbb{Z}^{6}$ & $(H, 8,15)$ & $\left(H^{\prime}, 7,15\right)$ \\
\cline { 2 - 7 } & $4 T$ & 2 & 6 & $\mathbb{Z}^{8}$ & $(H, 12,21)$ & $\left(H^{\prime}, 9,21\right)$ \\
\hline \multirow{6}{*}{$\tilde{5} P$} & $2 P$ & 8 & 1 & $\mathbb{Z}_{2} \oplus \mathbb{Z}$ & $(H, 2,3)$ & $\left(H^{\prime}, 1,3\right)$ \\
\cline { 2 - 7 } & $3 P$ & 7 & 2 & $\mathbb{Z}_{2} \oplus \mathbb{Z}^{2}$ & $(H, 4,6)$ & $\left(H^{\prime}, 3,6\right)$ \\
\cline { 2 - 7 } & $4 P$ & 6 & 4 & $\mathbb{Z}_{2} \oplus \mathbb{Z}^{3}$ & $(H, 6,9)$ & $\left(H^{\prime}, 3,9\right)$ \\
\cline { 2 - 7 } & $5 P$ & 5 & 10 & $\mathbb{Z}_{2} \oplus \mathbb{Z}^{4}$ & $(H, 8,12)$ & $\left(H^{\prime}, 4,12\right)$ \\
\cline { 2 - 7 } & $6 P$ & 4 & 13 & $\mathbb{Z}_{2} \oplus \mathbb{Z}^{5}$ & $(H, 10,15)$ & $\left(H^{\prime}, 5,15\right)$ \\
\cline { 2 - 7 } & $7 P$ & 3 & 14 & $\mathbb{Z}_{2} \oplus \mathbb{Z}^{6}$ & $(H, 12,18)$ & $\left(H^{\prime}, 6,18\right)$ \\
\cline { 2 - 7 } & $8 P$ & 2 & 6 & $\mathbb{Z}_{2} \oplus \mathbb{Z}^{7}$ & $(H, 14,21)$ & $\left(H^{\prime}, 7,21\right)$ \\
\cline { 2 - 7 } & $9 P$ & 1 & 1 & $\mathbb{Z}_{2} \oplus \mathbb{Z}^{8}$ & $(H, 16,24)$ & $\left(H^{\prime}, 6,24\right)$ \\
\hline
\end{tabular}

Table 3 - IS components coming from $K_{4,4}$ embeddings. 
By equalities (4) and (1), we have

$$
\gamma\left(K_{4,4}\right)=\left\{\frac{1}{4}(4-2)(4-2)=1 \text { and } \gamma_{M}\left(K_{4,4}\right)=\left[\frac{(4-1)(4-1)}{4}\right]\right\}=4 .
$$

Then, the orientable embedding surface sets is $\mathbb{S}\left(K_{4,4}\right)=\{T, 2 T, 3 T\}$, nonorientable embedding surface sets is $\mathbb{S}\left(K_{4,4}\right)=\{2 P, 3 P, \cdots, 8 P\}$ ( $2^{\text {nd }}$ column $)$ and the these partitions are related in the $3^{\text {rd }}$ column. The $4^{\text {th }}$ column indicates the number $\alpha$ of the regions which it is constant in each surface. In the last three columns are the structures of compatible codes.

The generalized codes on the sphere and torus are degenerated cases. By Theorem 5.4, only dual code is defined on the torus. Indeed, generalized codes are more appropriated for surfaces of large genus. As $H_{1}(S)=0$ the sphere is the unique case where the code coming from homology group is degenerated.

\section{Conclusion}

The integrated system is an ambitious idea, which intends to integrate the three main components of the data transmission system: codification, modulation and channel. In this first approach, the goal is to establish mathematic definitions considering the dependence relations of these components. In principle, the aim is to design a new data transmission system more efficient than the currently used. However, many other advantages are observed. The topological invariants such as orientability, genus, border component and partition, provide quick answers to questions about the modulation efficiency. It is not different with homology group. It is an important algebraic invariant which gives quick answers related to probably code class that could be projected in metrical spaces, such as Riemannian manifolds. The initial project, the topological constructing and identification of IS components, already has been solved in this work. The final project, has found in Algebraic Topology environments and Riemann's Geometry, recognized as promising areas for the communication problem. For further works, the proposal for the suggestions is to development embedding methods, constructing modulations that have been identified in this work (or new projects) using Riemann's Geometry tools and Algebraic Topology, and using the invariants as efficiency analysis instruments of the data transmission system. 
Acknowledgements. Our warmest thanks go to Dr. Reginaldo Palazzo Jr., from State University of Campinas - Brazil, as well as to the anonymous reviewers for their important comments and suggestions.

\section{REFERENCES}

[1] R.G. Cavalcante, H. Lazary, J.D. Lima and R. Palazzo Jr., A new approach to the design of digital communication systems. Series in Discret Mathematics an Theorical Computer Science, (2005), 145-177.

[2] R.G. Cavalcante, H. Lazary, J.D. Lima and R. Palazzo Jr., Coset space approach for the design of geometrically uniform codes in homogeneous spaces. In: XVIII Escola de Álgebra, 2004, Campinas. Anais da XVIII Escola de Álgebra. Campinas, (2005), 1-26.

[3] R.A. Duck, The genus, regional number, and Betti number of a graph. Canad. J. Math., 18 (1966), 817-822.

[4] W. Dyck, Reitrage zur Analysis Situs. Math. Ann., 32 (1888), 457-512.

[5] J.D. Lima and E.V. Matias, Modulation for signal constellations on Riemannian manifolds: associated channel and performance measure. Master Dissertation, Computer Science Mester, University of the State of the Rio Grande do Norte, Brazil, (2011). (in Portuguese).

[6] J.D. Lima, L.P.R.C. Lima and W.C.D. de Melo, Modulation for large signal constellations comming from embedding of complete graph, in ITS 2010 - VII International Telecomunications Symposium, 2010, Manaus - AM. Proceedings of ITS 2010 - International Telecommunications Symposim. Rio de Janeiro - RJ: Sociedade Brasileira de Telecomunicações, 32 (2010), 1-5.

[7] L.P.R.C. Lima, Modulation projects on topological surface associated to the discrete memoryless channels: a solution for the integrated system. Master Dissertation, Computer Science Mester, University of the State of the Rio Grande do Norte, Brazil, (2009). (in Portuguese).

[8] J.D. Lima, Identification and algebraic structure of compact surfaces with and without borders derived from embedding of discrete memoryless channels. Doctoral Dissertation, School of Electrical and Computer Engineering, State University of Campinas, Brazil, (2002), (in Portuguese).

[9] H.M. Oliveira, D.R. Oliveira and R.M. Souza, A unit quaternion-based spherical trigonometry and a new two-carrier phasequadrature quaternion modulation 
system. XXIX Simpósio Brasileiro de Telecomunicações - SBrT 2011, Curitiba, Paraná, (2011), 0-5.

[10] R.D. Reigensen, Determining all compact orientable 2-manifolds upon which $K_{m, n}$ has 2-cell embeddings. J. Combinatorial Theory, 12 (1972), 101-104.

[11] G. Ringuel, Das Geschlecht des vollständigen paaren Graphen. Math. Sem. Univ. Hamburg, 28 (1995), 139-150.

[12] J.W.T. Youngs and F. Ciryl, Minimal imbeddings and the genus of a graph. J. Math. Mech., 12 (1963), 303-316.

[13] J.M. Wozencraft and I.M. Jacobs, Principles of Comunication Engineering, $1^{\text {st }}$ Edition, New York, (1966).

[14] Y. Wang, F. Alajaji and T. Linder, Hybrid digital-analog coding with bandwidth compression for Gaussian source-channel pairs. IEEE Transactions on Communications, 57 (2009), 997-1012. 